\title{
On the Hybrid Fourth Power Mean Involving Legendre's Symbol and One Kind Two-Term Exponential Sums
}

\author{
Jingzhe Wang (iD) \\ School of Statistics and Mathematics, Inner Mongolia University of Finance and Economics, Hohhot 010070, \\ Inner Mongolia, China
}

Correspondence should be addressed to Jingzhe Wang; wangjingzhe729@126.com

Received 7 February 2021; Revised 24 February 2021; Accepted 26 February 2021; Published 11 March 2021

Academic Editor: Tingting Wang

Copyright ( 92021 Jingzhe Wang. This is an open access article distributed under the Creative Commons Attribution License, which permits unrestricted use, distribution, and reproduction in any medium, provided the original work is properly cited.

The main purpose of this article is using the elementary methods and the properties of the quadratic residue modulo an odd prime $p$ to study the calculating problem of the fourth power mean of one kind two-term exponential sums and give an interesting calculating formula for it.

\section{Introduction}

Let $q \geq 3$ be a fixed integer. For any integer $k \geq 2$ and integer $m$ with $(m, q)=1$, we define the two-term exponential sums $G(m, k ; q)$ as follows:

$$
G(m, k ; q)=\sum_{a=0}^{q-1} e\left(\frac{m a^{k}+a}{q}\right)
$$

where, as usual, $e(y)=e^{2 \pi i y}$ and $i$ denotes the imaginary unit, that is $i^{2}=-1$.

Since this kind of sums play a very important role in the study of analytic number theory, so many number theorists and scholars had studied the various properties of $G(m, k ; q)$ and obtained a series of meaningful research results, we do not want to enumerate here, and interested readers can refer to [1-16]. For example, Zhang and Zhang [1] proved that for any odd prime $p$, one has

$$
\sum_{m=1}^{p-1}\left|\sum_{a=0}^{p-1} e\left(\frac{m a^{3}+n a}{p}\right)\right|^{4}= \begin{cases}2 p^{3}-p^{2}, & \text { if } 3+p-1, \\ 2 p^{3}-7 p^{2}, & \text { if } 3 \mid p-1,\end{cases}
$$

where $n$ represents any integer with $(n, p)=1$.

Shen and Zhang [2] obtained an interesting recurrence formula for

$$
A(m, 4 ; p)=\sum_{a=0}^{p-1} e\left(\frac{m a^{4}}{p}\right)
$$

where $p$ is an odd prime with $p \equiv 1 \bmod 4$.

Chen and Zhang [3] proved that for any prime $p$ with $p \equiv 5 \bmod 8$, one has the identity

$$
\sum_{m=1}^{p-1}\left|\sum_{a=0}^{p-1} e\left(\frac{m a^{4}}{p}\right)\right|^{2} \cdot\left|\sum_{a=1}^{p-1} e\left(\frac{m a+\bar{a}}{p}\right)\right|^{2}=3 p^{3}-3 p^{2}+2 p^{(3 / 2)} \alpha-3 p,
$$

where $\alpha=\alpha(p)=\sum_{a=1}^{(p-1 / 2)}(a+\bar{a} / p),(* / p)$ denotes Legendre's symbol modulo $p$, and $a \cdot \bar{a} \equiv 1 \bmod p$.

Zhang and Han [4] used the elementary method to obtain the identity

$$
\sum_{a=1}^{p-1}\left|\sum_{n=0}^{p-1} e\left(\frac{n^{3}+a n}{p}\right)\right|^{6}=5 p^{4}-8 p^{3}-p^{2}
$$

where $p$ denotes an odd prime with $3+(p-1)$.

Chen and Wang [5] studied the calculating problem of the fourth power mean of $G(m, 4 ; p)$ and proved the following conclusion.

Let $p>3$ be an odd prime, then one has the identity 


$$
\sum_{m=1}^{p-1}\left|\sum_{a=0}^{p-1} e\left(\frac{m a^{4}+a}{p}\right)\right|^{4}= \begin{cases}2 p^{3}, & \text { if } p=12 k+11 \\ 2 p^{2}(p-2), & \text { if } p=12 k+7 \\ 2 p\left(p^{2}-4 p-2 \alpha^{2}\right), & \text { if } p=24 k+5 \\ 2 p\left(p^{2}-6 p-2 \alpha^{2}\right), & \text { if } p=24 k+13 \\ 2 p\left(p^{2}-10 p-2 \alpha^{2}\right), & \text { if } p=24 k+1 \\ 2 p\left(p^{2}-8 p-2 \alpha^{2}\right), & \text { if } p=24 k+17\end{cases}
$$

where $\alpha=\sum_{a=1}^{(p-1 / 2)}(a+\bar{a} / p)$ and $(* / p)$ denotes Legendre's symbol modulo $p$.

Zhang and Zhang [6] proved that for any prime $p$, one has the identity

$$
\sum_{m=1}^{p-1}\left(\frac{m}{p}\right) \cdot\left|\sum_{a=0}^{p-1} e\left(\frac{m a^{3}+a}{p}\right)\right|^{4}= \begin{cases}p^{2}(\delta-3), & \text { if } p \equiv 1 \bmod 6 \\ p^{2}(\delta+3), & \text { if } p \equiv-1 \bmod 6\end{cases}
$$

where $\delta=\sum_{d=1}^{p-1}(d-1+\bar{d} / p)$ is an integer which satisfies the estimate $|\delta| \leq 2 \sqrt{p}$.
Liu and Zhang [7] proved that for any prime $p$ with $3+(p-1)$, one has the identity

$$
\sum_{\chi \bmod } \sum_{p}^{p-1}\left|\sum_{m=0}^{p-1} \chi(a) e\left(\frac{m a^{3}+a}{p}\right)\right|^{6}=p(p-1)\left(6 p^{3}-28 p^{2}+39 p+5\right)
$$

where $\sum_{\chi \bmod p}$ denotes the summation over all Dirichlet characters, modulo $p$.

Inspired by the works in $[5,6]$, in this paper, we consider the following calculating problem of the $2 h$-th power mean of the two-term exponential sums:

$$
\sum_{m=1}^{p-1}\left(\frac{m}{p}\right) \cdot\left|\sum_{a=0}^{p-1} e\left(\frac{m a^{4}+n a}{p}\right)\right|^{2 h}
$$

where $p$ is an odd prime and $h \geq 2$ is an integer.
About this problem, it seems that none had studied it before; at least we have not seen such a result at present. In this paper, we will use the properties of the solutions of the congruence equations and the quadratic residue to study this problem and give an interesting calculating formula for (9) with $h=2$. That is, we will prove the following result.

Theorem 1. Let $p>3$ be an odd prime, then we have the identity

$$
\sum_{m=1}^{p-1}\left(\frac{m}{p}\right) \cdot\left|\sum_{a=0}^{p-1} e\left(\frac{m a^{4}+a}{p}\right)\right|^{4}= \begin{cases}2 p^{(3 / 2)}\left(6 \alpha-\left(\frac{3}{p}\right) \cdot p\right), & \text { if } p=8 k+5 \\ 2 p^{(3 / 2)}\left(\left(\frac{3}{p}\right) \cdot p-10 \alpha\right), & \text { if } p=8 k+1 \\ 0, & \text { if } p=4 k+3\end{cases}
$$

where $\alpha=\sum_{a=1}^{(p-1 / 2)}(a+\bar{a} / p)$ and $(* / p)$ denotes Legendre's symbol modulo $p$.

Note that the estimate $|\alpha| \leq \sqrt{p}$ (see [17] for general results), and from this theorem and [5], we may immediately deduce the following several corollaries.
Corollary 1. Let $p>3$ be an odd prime with $p \equiv 1 \bmod 4$, then we have the asymptotic formula

$$
\sum_{m=1}^{p-1}\left(\frac{m}{p}\right) \cdot\left|\sum_{a=0}^{p-1} e\left(\frac{m a^{4}+a}{p}\right)\right|^{4}=2 \cdot\left(\frac{6}{p}\right) \cdot p^{(5 / 2)}+O\left(p^{2}\right) .
$$


Corollary 2. Let $p>3$ be an odd prime with $p \equiv 1 \bmod 4$, then we have the asymptotic formula

$$
\sum_{m=1}^{p-1}\left|\sum_{a=0}^{p-1} e\left(\frac{m^{2} a^{4}+a}{p}\right)\right|^{4}=2 p^{3}+2 \cdot\left(\frac{6}{p}\right) \cdot p^{(5 / 2)}+O\left(p^{2}\right) \text {. }
$$

Some notes: the constant $\alpha=\alpha(p)$ in our theorem has a special meaning. In fact, for any prime $p$ with $p \equiv 1 \bmod 4$, one has the identity (see [18])

$$
p=\left(\frac{1}{2} \sum_{a=1}^{p-1}\left(\frac{a+\bar{a}}{p}\right)\right)^{2}+\left(\frac{1}{2} \sum_{a=1}^{p-1}\left(\frac{a+r \bar{a}}{p}\right)\right)^{2}=\alpha^{2}+\beta^{2}
$$

where $r$ is any quadratic nonresidue modulo $p$. That is, $(r / p)=-1$.

For any prime $p=4 k+1$ and integer $h \geq 3$, we naturally ask whether there is an exact calculating formula for (9)?

This is an open problem. We believe this to be true. We even have the following.

Conjecture 1. Let $p$ be an odd prime with $p \equiv 1 \bmod 4$. Then, for any integer $h \geq 2$, there are two integers $C=C(h, p)$ and $D=D(h, p)$ depending only on $h$ and $p$, such that the identity

$$
\sum_{m=1}^{p-1}\left(\frac{m}{p}\right) \cdot\left|\sum_{a=0}^{p-1} e\left(\frac{m a^{4}+a}{p}\right)\right|^{2 h}=C \cdot p^{(2 h+1 / 2)}+D \cdot p^{(2 h-1 / 2)} \cdot \alpha .
$$

\section{Several Lemmas}

In this section, we will give several necessary lemmas. Of course, the proofs of some lemmas need the knowledge of elementary and analytic number theory. In particular, the properties of the quadratic residues and the Legendre's symbol modulo $p$ are going to be used. All these can be found in $[15,18-20]$, and we do not repeat them. First, we have the following lemma.

Lemma 1. Let $p$ be an odd prime with $p \equiv 1 \bmod 4$. Then, for any fourth-order character $\lambda \bmod p$, we have the identity

$$
\tau^{2}(\lambda)+\tau^{2}(\bar{\lambda})=2 \sqrt{p} \cdot \alpha
$$

where $\tau(\chi)=\sum_{a=1}^{p-1} \chi(a) e(a / p)$ denotes the classical Gauss sums, $\alpha=\alpha(p)=\sum_{a=1}^{(p-1 / 2)}(a+\bar{a} / p)$, and $(* / p)=\chi_{2}$ denotes Legendre's symbol modulo $p$.

Proof. See Lemma 2 in Chen and Zhang [3].

Lemma 2. Let $p$ be an odd prime with $p \equiv 1 \bmod 4$, then we have the identity

$$
\sum_{a=0}^{p-1} \sum_{b=0}^{p-1} \sum_{c=0}^{p-1} \chi_{2}\left(a^{4}+b^{4}-c^{4}-1\right)= \begin{cases}-p \cdot(12 \alpha+3), & \text { if } p=8 k+5 \\ p \cdot(20 \alpha-7), & \text { if } p=8 k+1,\end{cases}
$$

where $\alpha=\alpha(p)$ is defined as in Lemma 1.

Proof. For any odd prime $p$ with $p \equiv 1 \bmod 4$, let $\lambda$ be any fourth-order character modulo $p$, and

$$
A(m)=\sum_{a=0}^{p-1} e\left(\frac{m a^{4}}{p}\right)
$$
have

Then, from the properties of the classical Gauss sums, we

$$
\begin{aligned}
A(m) & =1+\sum_{a=1}^{p-1}\left(1+\lambda(a)+\chi_{2}(a)+\bar{\lambda}(a)\right) \cdot e\left(\frac{m a}{p}\right) \\
& =\bar{\lambda}(m) \cdot \tau(\lambda)+\chi_{2}(m) \cdot \tau\left(\chi_{2}\right)+\lambda(m) \cdot \tau(\bar{\lambda}),
\end{aligned}
$$

where $(m, p)=1$. Note that $\tau\left(\chi_{2}\right)=\sqrt{p}, \chi_{2} \cdot \lambda=\bar{\lambda}$, from (18) and Lemma 1, we have

$$
\begin{aligned}
A^{2}(m) & =\left(\bar{\lambda}(m) \cdot \tau(\lambda)+\chi_{2}(m) \cdot \tau\left(\chi_{2}\right)+\lambda(m) \cdot \tau(\bar{\lambda})\right)^{2} \\
& =p+2 \sqrt{p} \cdot(\lambda(m) \cdot \tau(\lambda)+\bar{\lambda}(m) \cdot \tau(\bar{\lambda}))+2 \tau(\lambda) \cdot \tau(\bar{\lambda})+\chi_{2}(m) \cdot 2 \cdot \sqrt{p} \cdot \alpha,
\end{aligned}
$$

where $p \equiv 1 \bmod 4$. If $p=8 k+5$, then $\lambda(-1)=-1$ and $\tau(\lambda) \cdot \tau(\bar{\lambda})=-p$, so we have

$$
\begin{aligned}
A^{2}(m) \cdot A(-m) & =\left(-p+2 \sqrt{p} \cdot(\lambda(m) \cdot \tau(\lambda)+\bar{\lambda}(m) \cdot \tau(\bar{\lambda}))+\chi_{2}(m) \cdot 2 \cdot \sqrt{p} \cdot \alpha\right) \times\left(-\bar{\lambda}(m) \cdot \tau(\lambda)+\chi_{2}(m) \cdot \sqrt{p}-\lambda(m) \cdot \tau(\bar{\lambda})\right) \\
& =3 \chi_{2}(m) p^{(3 / 2)}+3 p(\bar{\lambda}(m) \tau(\lambda)+\lambda(m) \tau(\bar{\lambda}))-2 \sqrt{p} \alpha(\lambda(m) \tau(\lambda)+\bar{\lambda}(m) \tau(\bar{\lambda}))-2 p \alpha .
\end{aligned}
$$
have

If $p=8 k+1$, then $\lambda(-1)=1$ and $\tau(\lambda) \cdot \tau(\bar{\lambda})=p$, so we 


$$
\begin{aligned}
A^{2}(m) \cdot A(-m) & =\left(3 p+2 \sqrt{p} \cdot(\lambda(m) \cdot \tau(\lambda)+\bar{\lambda}(m) \cdot \tau(\bar{\lambda}))+\chi_{2}(m) \cdot 2 \cdot \sqrt{p} \cdot \alpha\right) \times\left(\bar{\lambda}(m) \cdot \tau(\lambda)+\chi_{2}(m) \cdot \sqrt{p}+\lambda(m) \cdot \tau(\bar{\lambda})\right) \\
& =7 \chi_{2}(m) p^{(3 / 2)}+5 p(\bar{\lambda}(m) \tau(\lambda)+\lambda(m) \tau(\bar{\lambda}))+6 p \alpha+2 \sqrt{p} \alpha(\lambda(m) \tau(\lambda)+\bar{\lambda}(m) \tau(\bar{\lambda})) .
\end{aligned}
$$

Now, if $p=8 k+5$, then from (20) and Lemma 1, we have

$$
\begin{aligned}
\tau\left(\chi_{2}\right) \sum_{a=0}^{p-1} \sum_{b=0}^{p-1} \sum_{c=0}^{p-1} \chi_{2}\left(a^{4}+b^{4}-c^{4}-1\right) \\
=\sum_{m=1}^{p-1} \chi_{2}(m) \cdot A^{2}(m) \cdot A(-m) \cdot e\left(\frac{-m}{p}\right) \\
=-3 p^{(3 / 2)}+3 p \cdot \sum_{m=1}^{p-1}(\lambda(m) \tau(\lambda)+\bar{\lambda}(m) \tau(\bar{\lambda})) \cdot e\left(\frac{-m}{p}\right) \\
\quad-2 \sqrt{p} \alpha \cdot \sum_{m=1}^{p-1}(\bar{\lambda}(m) \tau(\lambda)+\lambda(m) \tau(\bar{\lambda})) \cdot e\left(\frac{-m}{p}\right)-2 p^{(3 / 2)} \cdot \alpha \\
=-3 p^{(3 / 2)}-3 p \cdot\left(\tau^{2}(\lambda)+\tau^{2}(\bar{\lambda})\right)-4 p^{(3 / 2)} \cdot \alpha-2 p^{(3 / 2)} \cdot \alpha \\
=-p^{(3 / 2)} \cdot(12 \alpha+3) .
\end{aligned}
$$

If $p=8 k+1$, then from (21) and Lemma 1 , we have

$$
\begin{aligned}
\tau\left(\chi_{2}\right) \sum_{a=0}^{p-1} \sum_{b=0}^{p-1} \sum_{c=0}^{p-1} \chi_{2}\left(a^{4}+b^{4}-c^{4}-1\right) \\
=\sum_{m=1}^{p-1} \chi_{2}(m) \cdot A^{2}(m) \cdot A(-m) \cdot e\left(\frac{-m}{p}\right) \\
=-7 p^{(3 / 2)}+5 p \cdot \sum_{m=1}^{p-1}(\lambda(m) \tau(\lambda)+\bar{\lambda}(m) \tau(\bar{\lambda})) \cdot e\left(\frac{-m}{p}\right) \\
\quad+2 \sqrt{p} \alpha \cdot \sum_{m=1}^{p-1}(\bar{\lambda}(m) \tau(\lambda)+\lambda(m) \tau(\bar{\lambda})) \cdot e\left(\frac{-m}{p}\right)+6 p^{(3 / 2)} \cdot \alpha \\
=-7 p^{(3 / 2)}+5 p \cdot\left(\tau^{2}(\lambda)+\tau^{2}(\bar{\lambda})\right)+4 p^{(3 / 2)} \cdot \alpha+6 p^{(3 / 2)} \cdot \alpha \\
=p^{(3 / 2)} \cdot(20 \cdot \alpha-7) .
\end{aligned}
$$

Combining (22) and (23) and $\tau\left(\chi_{2}\right)=\sqrt{p}$, we may immediately deduce Lemma 2.

Lemma 3. Let $p$ be an odd prime with $p \equiv 1 \bmod 4$. Then, we have the identity

$$
\sum_{a=0}^{p-1} \sum_{b=0}^{p-1} \chi_{2}\left(a^{4}+1-b^{4}\right)= \begin{cases}3 p+2 \alpha, & \text { if } p=8 k+5 \\ 7 p-2 \alpha, & \text { if } p=8 k+1 .\end{cases}
$$

Proof. From the methods of proving Lemma 2, we have

$$
\sum_{a=0}^{p-1} \sum_{b=0}^{p-1} \chi_{2}\left(a^{4}+1-b^{4}\right)=\frac{1}{\sqrt{p}} \cdot \sum_{m=1}^{p-1} \chi_{2}(m)|A(m)|^{2} \cdot e\left(\frac{m}{p}\right) .
$$

If $p=8 k+5$, then note that $\overline{\tau(\lambda)}=-\tau(\bar{\lambda})$, and from (18), we have

$$
|A(m)|^{2}=3 p-\chi_{2}(m) \cdot\left(\tau^{2}(\lambda)+\tau^{2}(\bar{\lambda})\right)=3 p-2 \chi_{2}(m) \cdot \sqrt{p} \cdot \alpha,
$$

Then, applying (25), we have

$$
\begin{aligned}
\sum_{a=0}^{p-1} \sum_{b=0}^{p-1} \chi_{2}\left(a^{4}+1-b^{4}\right)= & \frac{1}{\sqrt{p}} \cdot \sum_{m=1}^{p-1} \chi_{2}(m)\left(3 p-2 \chi_{2}(m)\right. \\
& \cdot \sqrt{p} \cdot \alpha) \cdot e\left(\frac{m}{p}\right) \\
= & 3 p+2 \alpha .
\end{aligned}
$$

If $p=8 k+1$, then $|A(m)|^{2}=A^{2}(m)$, and from (19) and (25), we have

$$
\begin{aligned}
& \sum_{a=0}^{p-1} \sum_{b=0}^{p-1} \chi_{2}\left(a^{4}+1-b^{4}\right)=\frac{1}{\sqrt{p}} \cdot \sum_{m=1}^{p-1} \chi_{2}(m)|A(m)|^{2} \cdot e\left(\frac{m}{p}\right) \\
& \quad=\sum_{m=1}^{p-1} \chi_{2}(m)\left(3 \sqrt{p}+2 \cdot(\lambda(m) \cdot \tau(\lambda)+\bar{\lambda}(m) \cdot \tau(\bar{\lambda}))+\chi_{2}(m) \cdot 2 \cdot \alpha\right) \cdot e\left(\frac{m}{p}\right) \\
& \quad=7 p-2 \alpha .
\end{aligned}
$$

Now, Lemma 3 follows from (27) and (28). 
Lemma 4. Let $p>3$ be a prime with $p \equiv 1 \bmod 4$. Then, we have the identity

$$
\begin{aligned}
& \sum_{\substack{a=0 \\
a+b \equiv c+1 \bmod p}}^{p-1} \sum_{b=0}^{p-1} \sum_{c=0}^{p-1} \chi_{2}\left(a^{4}+b^{4}-c^{4}-1\right)=2 \cdot \chi_{2}(6) \cdot p-\chi_{2}(2) \\
& \quad \cdot \sum_{b=0}^{p-1} \chi_{2}\left(b^{3}+b+2\right) .
\end{aligned}
$$

Proof. From the properties of the complete residue system modulo $p$, we have

$$
\begin{aligned}
& \sum_{a=0}^{p-1} \sum_{b=0}^{p-1} \sum_{c=0}^{p-1} \chi_{2}\left(a^{4}+b^{4}-c^{4}-1\right) \\
& a+b \equiv c+1 \bmod p \\
& =\sum_{\substack{a=0 \\
a+b=0}}^{p-1} \sum_{\substack{b=0 \\
a+b}}^{p-1} \sum_{\substack{c-1 \\
\text { mod } p}}\left((a+c)^{4}+(b+1)^{4}-c^{4}-1\right) \\
& =\sum_{a=0}^{p-1} \sum_{c=0}^{p-1} \chi_{2}\left(a^{4}+4 a^{3} c+6 a^{2} c^{2}+4 a c^{3}+a^{4}-4 a^{3}+6 a^{2}-4 a\right) \\
& =\sum_{a=1}^{p-1} \sum_{c=0}^{p-1} \chi_{2}\left(a^{4}+4 a^{4} c+6 a^{4} c^{2}+4 a^{4} c^{3}+a^{4}-4 a^{3}+6 a^{2}-4 a\right) \\
& =\sum_{a=1}^{p-1} \sum_{c=0}^{p-1} \chi_{2}\left(2+4 c+6 c^{2}+4 c^{3}-4 a+6 a^{2}-4 a^{3}\right) \\
& =\chi_{2}(2) \cdot \sum_{a=1}^{p-1} \sum_{c=0}^{p-1} \chi_{2}\left(4+4 c+3 c^{2}+c^{3}-4 a+3 a^{2}-a^{3}\right) \\
& =\chi_{2}(2) \cdot \sum_{a=1}^{p-1} \sum_{c=0}^{p-1} \chi_{2}\left((c+1)^{3}+c+1-(a-1)^{3}-(a-1)\right) \\
& =\chi_{2}(2) \cdot \sum_{a=0}^{p-1} \sum_{c=0}^{p-1} \chi_{2}\left(c^{3}+c-a^{3}-a\right)-\chi_{2}(2) \cdot \sum_{b=0}^{p-1} \chi_{2}\left(b^{3}+b+2\right) \\
& =\chi_{2}(2) \cdot \sum_{a=0}^{p-1} \sum_{c=0}^{p-1} \chi_{2}\left(c^{3}+3 c^{2} a+3 c a^{2}+c\right)-\chi_{2}(2) \cdot \sum_{b=0}^{p-1} \chi_{2}\left(b^{3}+b+2\right) \\
& =\chi_{2}(2) \cdot \sum_{a=0}^{p-1} \sum_{c=0}^{p-1} \chi_{2}\left(c^{3}+3 c(2 a+c)^{2}+4 c\right)-\chi_{2}(2) \\
& \cdot \sum_{b=0}^{p-1} \chi_{2}\left(b^{3}+b+2\right) \\
& =\chi_{2}(2) \cdot \sum_{a=0}^{p-1} \sum_{c=1}^{p-1} \chi_{2}\left(c^{3}+3 c a^{2}+4 c\right)-\chi_{2}(2) \cdot \sum_{b=0}^{p-1} \chi_{2}\left(b^{3}+b+2\right) \text {. }
\end{aligned}
$$

Note that the identity

$$
\sum_{a=0}^{p-1}\left(\frac{a^{2}+n}{p}\right)= \begin{cases}p-1, & \text { if }(n, p)=p \\ -1, & \text { if }(n, p)=1,\end{cases}
$$

We have

and $\chi_{2}(2)=-1$, if $p=8 k+5 ; \chi_{2}(2)=1$, if $p=8 k+1$.

$$
\begin{aligned}
\sum_{a=0}^{p-1} \sum_{c=1}^{p-1} \chi_{2}\left(c^{3}+3 c a^{2}+4 c\right) & =p \cdot \sum_{\substack{c=1 \\
c^{3}+4 c \equiv 0 \bmod p}}^{p-1} \chi_{2}(3 c)-\sum_{c=1}^{p-1} \chi_{2}(3 c) \\
& =p \cdot \sum_{\substack{c=1 \\
c^{2}+1 \equiv 0 \bmod p}}^{p-1} \chi_{2}(6 c)=2 \cdot \chi_{2}(3) \cdot p
\end{aligned}
$$

From (30) and (32), we have

$$
\begin{aligned}
& \sum_{\substack{a=0 \\
a+b \equiv c}}^{p-1} \sum_{b=0}^{p-1} \sum_{c=0}^{p-1} \chi_{2}\left(a^{4}+b^{4}-c^{4}-1\right)=2 \cdot \chi_{2}(6) \cdot p-\chi_{2}(2) \\
& \quad \cdot \sum_{b=0}^{p-1} \chi_{2}\left(b^{3}+b+2\right)
\end{aligned}
$$

This proves Lemma 4.

Lemma 5. Let $p>3$ be a prime with $p \equiv 1 \bmod 4$. Then, we have the identity

$$
\sum_{a=0}^{p-1} \sum_{b=0}^{p-1} \sum_{c=0}^{p-1} \chi_{2}\left(a^{4}+b^{4}-c^{4}\right) e\left(\frac{a+b-c}{p}\right)=\left\{\begin{array}{cc}
\chi_{2}(2) \cdot p \cdot \sum_{b=0}^{p-1} \chi_{2}\left(b^{3}+b+2\right)-3 p, & \text { if } p=8 k+5 \\
\chi_{2}(2) \cdot p \cdot \sum_{b=0}^{p-1} \chi_{2}\left(b^{3}+b+2\right)-7 p, & \text { if } p=8 k+1 .
\end{array}\right.
$$


Proof. It is clear that

$$
\begin{aligned}
& \sum_{a=0}^{p-1} \sum_{b=0}^{p-1} \sum_{c=0}^{p-1} \chi_{2}\left(a^{4}+b^{4}-c^{4}\right) e\left(\frac{a+b-c}{p}\right) \\
& =\sum_{a=1}^{p-1} \sum_{b=0}^{p-1} \sum_{c=0}^{p-1} \chi_{2}\left(a^{4}+b^{4}-c^{4}\right) e\left(\frac{a+b-c}{p}\right)+\sum_{b=0}^{p-1} \sum_{c=0}^{p-1} \chi_{2}\left(b^{4}-c^{4}\right) e\left(\frac{b-c}{p}\right) \\
& =p \cdot \sum_{\substack{b=0 \\
b+1 \equiv c \bmod p}}^{p-1} \sum_{c=0}^{p-1} \chi_{2}\left(1+b^{4}-c^{4}\right)-\sum_{b=0}^{p-1} \sum_{c=0}^{p-1} \chi_{2}\left(1+b^{4}-c^{4}\right)-1 \\
& +\sum_{b=0}^{p-1} \chi_{2}\left(b^{4}-1\right) \sum_{c=0}^{p-1} e\left(\frac{c(b-1)}{p}\right)-\sum_{b=0}^{p-1} \chi_{2}\left(b^{4}-1\right) \\
& =p \cdot \sum_{b=0}^{p-1} \chi_{2}\left(1+b^{4}-(b+1)^{4}\right)-\sum_{b=0}^{p-1} \sum_{c=0}^{p-1} \chi_{2}\left(1+b^{4}-c^{4}\right)-1-\sum_{b=0}^{p-1} \chi_{2}\left(b^{4}-1\right) \\
& =p \cdot \sum_{b=0}^{p-1} \chi_{2}\left(4 b^{3}+6 b^{2}+4 b\right)-\sum_{b=0}^{p-1} \sum_{c=0}^{p-1} \chi_{2}\left(1+b^{4}-c^{4}\right)-1-\sum_{b=0}^{p-1} \chi_{2}\left(b^{4}-1\right) . \\
& \sum_{b=0}^{p-1} \chi_{2}\left(4 b^{3}+6 b^{2}+4 b\right)=\chi_{2}(4) \sum_{b=0}^{p-1} \chi_{2}\left(4 b^{3}+6 b^{2}+4 b\right) \\
& =\chi_{2}(2) \cdot \sum_{b=0}^{p-1} \chi_{2}\left((2 b)^{3}+3(2 b)^{2}+4(2 b)\right) \\
& =\chi_{2}(2) \cdot \sum_{b=0}^{p-1} \chi_{2}\left(b^{3}+3 b^{2}+4 b\right) \\
& =\chi_{2}(2) \cdot \sum_{b=0}^{p-1} \chi_{2}\left((b+1)^{3}+b+1-2\right) \\
& =\chi_{2}(2) \cdot \sum_{b=0}^{p-1} \chi_{2}\left(b^{3}+b-2\right) \\
& =\chi_{2}(2) \cdot \sum_{b=0}^{p-1} \chi_{2}\left(b^{3}+b+2\right) \text {. }
\end{aligned}
$$

From (31), we have

$$
\begin{aligned}
& \sum_{b=0}^{p-1} \chi_{2}\left(b^{4}-1\right)=\sum_{b=0}^{p-1}\left(1+\chi_{2}(b)\right) \cdot \chi_{2}\left(b^{2}-1\right) \\
& =\sum_{b=0}^{p-1} \chi_{2}\left(b^{2}-1\right)+\sum_{b=1}^{p-1} \chi_{2}(b) \cdot \chi_{2}\left(b^{2}-1\right) \\
& =-1+\sum_{b=1}^{p-1} \chi_{2}(b-\bar{b})=-1+2 \cdot(-1)^{(p-1 / 4)} \cdot \alpha .
\end{aligned}
$$

Note that $\chi_{2}(4)=1$ and $(2, p)=1$, and from the properties of complete residue system modulo $p$ and $\chi_{2}(-1)=1$, we have

Combining (35)-(37) and Lemma 3, we have

$$
\sum_{a=0}^{p-1} \sum_{b=0}^{p-1} \sum_{c=0}^{p-1} \chi_{2}\left(a^{4}+b^{4}-c^{4}\right) e\left(\frac{a+b-c}{p}\right)= \begin{cases}\chi_{2}(2) \cdot p \cdot \sum_{b=0}^{p-1} \chi_{2}\left(b^{3}+b+2\right)-3 p, & \text { if } p=8 k+5 \\ \chi_{2}(2) \cdot p \cdot \sum_{b=0}^{p-1} \chi_{2}\left(b^{3}+b+2\right)-7 p, & \text { if } p=8 k+1 .\end{cases}
$$

This proves Lemma 5. 


\section{Proof of the Theorem}

Applying several basic lemmas in Section 2, we can easily complete the proof of our theorem. In fact, for any odd prime $p>3$, if $p=4 k+3$, then from the properties of the reduced residue system modulo $p$, we have

$$
\begin{aligned}
\sum_{m=1}^{p-1}\left(\frac{m}{p}\right) \cdot\left|\sum_{a=0}^{p-1} e\left(\frac{m a^{4}+a}{p}\right)\right|^{4}=\sum_{m=1}^{p-1}\left(\frac{-m}{p}\right) \cdot\left|\sum_{a=0}^{p-1} e\left(\frac{-m a^{4}+a}{p}\right)\right|^{4} \\
=-\sum_{m=1}^{p-1}\left(\frac{m}{p}\right) \cdot\left|\sum_{a=0}^{p-1} e\left(\frac{-m a^{4}-a}{p}\right)\right|^{4}=-\sum_{m=1}^{p-1}\left(\frac{m}{p}\right) \cdot\left|\sum_{a=0}^{p-1} e\left(\frac{m a^{4}+a}{p}\right)\right|^{4} .
\end{aligned}
$$

So, in this case, we have the identity

If $p=4 k+1$, then we have

$$
\sum_{m=1}^{p-1}\left(\frac{m}{p}\right) \cdot\left|\sum_{a=0}^{p-1} e\left(\frac{m a^{4}+a}{p}\right)\right|^{4}=0 .
$$

$$
\begin{aligned}
\sum_{m=1}^{p-1}\left(\frac{m}{p}\right) \cdot\left|\sum_{a=0}^{p-1} e\left(\frac{m a^{4}+a}{p}\right)\right|^{4} & \\
= & \tau\left(\chi_{2}\right) \cdot \sum_{a=0}^{p-1} \sum_{b=0}^{p-1} \sum_{c=0}^{p-1} \sum_{d=0}^{p-1} \chi_{2}\left(a^{4}+b^{4}-c^{4}-d^{4}\right) e\left(\frac{a+b-c-d}{p}\right) \\
= & \tau\left(\chi_{2}\right) \cdot \sum_{a=0}^{p-1} \sum_{b=0}^{p-1} \sum_{c=0}^{p-1} \sum_{d=1}^{p-1} \chi_{2}\left(a^{4}+b^{4}-c^{4}-1\right) e\left(\frac{d(a+b-c-1)}{p}\right) \\
& +\tau\left(\chi_{2}\right) \cdot \sum_{a=0}^{p-1} \sum_{b=0}^{p-1} \sum_{c=0}^{p-1} \chi_{2}\left(a^{4}+b^{4}-c^{4}\right) e\left(\frac{a+b-c}{p}\right) \\
= & \tau\left(\chi_{2}\right) \cdot p \cdot \sum_{a=0}^{p-1} \sum_{b=0}^{p-1} \sum_{c=0}^{p-1} \chi_{2}\left(a^{4}+b^{4}-c^{4}-1\right) \\
& -\tau\left(\chi_{2}\right) \cdot \sum_{a=0}^{p-1} \sum_{b=0}^{p-1} \sum_{c=0}^{p-1} \chi_{2}\left(a^{4}+b^{4}-c^{4}-1\right) \\
& +\tau\left(\chi_{2}\right) \cdot \sum_{a=0}^{p-1} \sum_{b=0}^{p-1} \sum_{c=0}^{p-1} \chi_{2}\left(a^{4}+b^{4}-c^{4}\right) e\left(\frac{a+b-c}{p}\right) .
\end{aligned}
$$

If $p=8 k+5$, then note that $\chi_{2}(2)=-1$, and from (41), Lemma 2, Lemma 4, and Lemma 5, we may immediately deduce 


$$
\begin{aligned}
& \sum_{m=1}^{p-1}\left(\frac{m}{p}\right) \cdot\left|\sum_{a=0}^{p-1} e\left(\frac{m a^{4}+a}{p}\right)\right|^{4} \\
& \quad=p^{(3 / 2)} \cdot\left(2 \cdot \chi_{2}(6) \cdot p-\chi_{2}(2) \cdot \sum_{b=0}^{p-1} \chi_{2}\left(b^{3}+b+2\right)\right)+p^{(3 / 2)} \cdot(12 \alpha+3)+p^{(1 / 2)} \cdot\left(\chi_{2}(2) \cdot p \cdot \sum_{b=0}^{p-1} \chi_{2}\left(b^{3}+b+2\right)-3 p\right) \\
& \quad=2 \cdot p^{(3 / 2)} \cdot\left(6 \alpha-\left(\frac{3}{p}\right) \cdot p\right) .
\end{aligned}
$$

Similarly, if $p=8 k+1$, then note that $\chi_{2}(2)=1$, and from (41), Lemma 2, Lemma 4, and Lemma 5, we may immediately deduce

$$
\begin{aligned}
& \sum_{m=1}^{p-1}\left(\frac{m}{p}\right) \cdot\left|\sum_{a=0}^{p-1} e\left(\frac{m a^{4}+a}{p}\right)\right|^{4} \\
& \quad=p^{(3 / 2)} \cdot\left(2 \cdot \chi_{2}(6) \cdot p-\chi_{2}(2) \cdot \sum_{b=0}^{p-1} \chi_{2}\left(b^{3}+b+2\right)\right)-p^{(3 / 2)} \cdot(20 \alpha-7)+p^{(1 / 2)} \cdot\left(\chi_{2}(2) \cdot p \cdot \sum_{b=0}^{p-1} \chi_{2}\left(b^{3}+b+2\right)-7 p\right) \\
& \quad=2 \cdot p^{(3 / 2)} \cdot\left(\left(\frac{3}{p}\right) \cdot p-10 \alpha\right) .
\end{aligned}
$$

Now, the theorem follows from (40), (42), and (43). This completes the proof of our main result.

\section{Data Availability}

The data used to support the findings of this study are included within the article.

\section{Conflicts of Interest}

The author declares that there are no conflicts of interest regarding the publication of this paper.

\section{Acknowledgments}

This work was supported by the NSF (11862018) of People's Republic of China and NSF (2017BS0101) of Inner Mongolia of China.

\section{References}

[1] H. Zhang and W. P. Zhang, "The fourth power mean of twoterm exponential sums and its application," Mathematical Reports, vol. 19, pp. 75-81, 2017.

[2] S. M. Shen and W. P. Zhang, "On the quartic Gauss sums and their recurrence property," Advances in Difference Equations, vol. 2017, p. 43, 2017.
[3] Z. Y. Chen and W. P. Zhang, "On the fourth-order linear recurrence formula related to classical Gauss sums," Open Mathematics, vol. 15, no. 1, pp. 1251-1255, 2017.

[4] W. Zhang and D. Han, "On the sixth power mean of the twoterm exponential sums," Journal of Number Theory, vol. 136, pp. 403-413, 2014.

[5] L. Chen and X. Wang, "A new fourth power mean of two-term exponential sums," Open Mathematics, vol. 17, no. 1, pp. 407-414, 2019.

[6] J. Zhang and W. P. Zhang, "A certain two-term exponential sum and its fourth power means," AIMS Mathematics, vol. 5, no. 6, pp. 7500-7509, 2020.

[7] X. Liu and W. Zhang, "On the high-power mean of the generalized Gauss sums and Kloosterman sums," Mathematics, vol. 7, no. 10, p. 907, 2019.

[8] X. X. Li and J. Y. Hu, "The hybrid power mean quartic Gauss sums and Kloosterman sums," Open Mathematics, vol. 15, no. 1, pp. 151-156, 2017.

[9] D. Han, "A hybrid mean value involving two-term exponential sums and polynomial character sums," Czechoslovak Mathematical Journal, vol. 64, pp. 53-62, 2014.

[10] L. Chen and Z. Y. Chen, "Some new hybrid power mean formulae of trigonometric sums," Advances in Differences Equation, vol. 2020, p. 220, 2020.

[11] L. Chen and J. Y. Hu, "A linear recurrence formula involving cubic Gauss sums and Kloosterman sums," Acta Mathematica Sinica (Chinese Series), vol. 61, pp. 67-72, 2018.

[12] S. Chowla, J. Cowles, and M. Cowles, "On the number of zeros of diagonal cubic forms," Journal of Number Theory, vol. 9, no. 4, pp. 502-506, 1977. 
[13] W. P. Zhang and J. Y. Hu, "The number of solutions of the diagonal cubic congruence equation $\bmod p$," Mathematical Reports, vol. 20, pp. 70-76, 2018.

[14] W. P. Zhang and H. N. Liu, "On the general Gauss sums and their fourth power mean," Osaka Journal of Mathematics, vol. 42, pp. 189-199, 2005.

[15] J. Z. Wang and Y. K. Ma, "The hybrid power mean of the $k$-th Gauss sums and Kloosterman sums," Journal of Shaanxi Normal University (Natural Science Edition), vol. 45, pp. 5-7, 2017.

[16] B. C. Berndt and R. J. Evans, "The determination of Gauss sums," Bulletin of the American Mathematical Society, vol. 5, no. 2, pp. 107-130, 1981.

[17] A. Weil, Basic Number Theory, Springer-Verlag, New York, NY, USA, 1974.

[18] W. P. Zhang and H. L. Li, Elementary Number Theory, Shaanxi Normal University Press, Xi'an, China, 2013.

[19] T. M. Apostol, Introduction to Analytic Number Theory, Springer-Verlag, New York, NY, USA, 1976.

[20] K. Ireland and M. Rosen, A Classical Introduction to Modern Number Theory, Springer-Verlag, New York, NY, USA, 1982. 